\title{
Risk assessment in avalanche-prone areas
}

\author{
Massimiliano BARbOLini, ${ }^{1}$ Federica CAPPABIANCA, ${ }^{1}$ Fabrizio SAVI ${ }^{2}$ \\ ${ }^{1}$ Hydraulic and Environmental Engineering Department, University of Pavia, Via Ferrata 1, I-27100 Pavia, Italy \\ E-mail:massimiliano.barbolini@unipv.it \\ ${ }^{2}$ Department of Hydraulics, Transportation and Highways, University "La Sapienza" of Rome, Via Eudossiana 18, I-00184 Rome, Italy
}

\begin{abstract}
This paper addresses the problem of defining a proper method for formal risk analysis in avalanche-prone areas. In this study, risk is defined as the annual probability of being killed by an avalanche for someone living or working permanently in a building under a hazardous hillside. A new methodology to estimate the hazard component of avalanche risk based on the use of dynamic models is introduced. This approach seems to have some advantages over the current methods based on statistical analysis of historic avalanche data. The vulnerability component of risk is formulated as a function of avalanche velocity, according to previous formulations. However, given the lack of knowledge on how avalanche impact damages structures and causes fatalities, the effect on the resulting risk mapping of using different vulnerability relations is explored. The potential of the proposed approach for evaluating the residual risk after the implementation of defensive structural work is discussed.
\end{abstract}

\section{INTRODUGTION}

Formal risk analysis may be considered the best method for evaluating the danger people face from avalanches, and for introducing proper land-use regulation in endangered areas. Estimating avalanche risk gives the information in a manner that is easy to understand and interpret, and enables a comparison to be made with other types of naturally or artificially induced risk. Nevertheless, only recently have such approaches emerged for analyzing the effect of snow avalanches (Wilhelm, 1998; Jónasson and others, 1999; Keylock and others, 1999; Keylock and Barbolini, 2001), and, except for Iceland (Jóhannesson and Arnalds, 2001), none of the existing zoning techniques adopts the risk approach explicitly.

According to the Committee on Risk Assessment of the Working Group on Landslides of the International Union of Geological Sciences (IUGS, 1997), quantitative risk analysis involves expressing the risk as a function of the hazard, the elements at risk and the vulnerability, where these terms are defined in Table 1 according to the IUGS (1997) guidelines.

Before the risk can be measured, one must decide what unit to use. There are several possible definitions of this unit. One might measure the risk as the yearly expected value of property loss due to avalanches (economic risk, measured for instance in $€$ /year; see Wilhelm, 1998), or as the number of people expected to be killed annually by avalanches in the area concerned (measured in deaths/year; see Wilhelm, 1998). In the present study, in accordance with the proposal of Jónasson and others (1999), we measure the risk as the annual probability of being killed by an avalanche if one lives or works in a building under a hazardous hillside (individual risk). Therefore human life is considered to be the element at risk (according to Table 1). To make this definition workable, one must also specify the proportion of time spent inside the building. As a reference value, we calculate the individual risk based on the assumption that the person is present in the building $100 \%$ of the time, and refer to this as local risk. This is the same unit as the one chosen by Jónasson and others (1999) for avalanche risk mapping and by Evans and others (1997) for aeroplane crash risk. The actual risk for a person is only a fraction of the local risk, depending on the fraction of time he/she spends in the building (usually referred to as the exposure), which in turn depends on the age of the person and on the type of building (e.g. for children or old people at home, the exposure might be as high as $75 \%$, compared with about $30 \%$ for people at their place of work). If a typical degree of exposure is defined for different types of buildings (dwellings, commercial buildings, cottages, etc.), as well as an acceptable risk level, local risk can be used as a feasible base for regulating land use in avalanche-prone areas, as suggested by the current Icelandic legislation (Jóhannesson and Arnalds, 2001).

Within such an approach the vulnerability (according to Table 1) becomes the probability of death for a person inside

Table 1. Definitions of risk terminology following appendix 1 of IUGS (1997). The word "avalanche" has been substituted for "landslide"

\begin{tabular}{ll}
\hline Term & \multicolumn{1}{c}{ IUGS definition } \\
\hline Risk & $\begin{array}{l}\text { A measure of the probability and severity of an adverse } \\
\text { effect to health, property or environment } \\
\text { The probability that a particular avalanche will occur } \\
\text { within a given time } \\
\text { Element at risk }\end{array}$ \\
$\begin{array}{l}\text { The population, building and engineering works, eco- } \\
\text { nomic activities, public service utilities and infrastruc- } \\
\text { tures in the area potentially affected by avalanches }\end{array}$ \\
$\begin{array}{l}\text { The degree of loss to a given element or set of elements } \\
\text { within the area affected by avalanche(s). It is expressed } \\
\text { on a scale of } 0 \text { (no loss) to } 1 \text { (total loss). For property, the } \\
\text { loss will be the value of the property, and for persons it } \\
\text { will be the probability that a particular life (the element } \\
\text { at risk) will be lost, given the person(s) affected by the } \\
\text { avalanche }\end{array}$
\end{tabular}


a house hit by an avalanche. This probability, in the following indicated as $d$, depends on the type of building and on the avalanche dynamic characteristics. In the present study, vulnerability is defined assuming a given structure type and as a function of avalanche velocity. Accordingly, the fatality ratio should broadly correlate with the impact pressure with which the avalanche strikes the building, and, therefore, ultimately with the avalanche velocity. Many authors (Mellor, 1978; McClung and Schaerer, 1993; Keylock and Barbolini, 2001), at least for flowing avalanches hitting large obstacles (such as buildings), have proposed a relation between avalanche velocity $(v)$ and impact pressure $\left(p_{\text {imp. }}\right)$ of the type:

$$
p_{\text {imp. }}=\rho v^{2},
$$

where $\rho$ is the avalanche density. Equation (1) is also suggested for engineering design by the Swiss and Russian Guidelines (Salm and others, 1990; Bozhinskiy and Losev, 1998), and has been adopted in the present study with $\rho$ set to $300 \mathrm{~kg} \mathrm{~m}^{-3}$. Undoubtedly, lack of knowledge of how avalanche impact damages structures and causes fatalities is a major limitation on any current avalanche risk assessment. Therefore, the effect of using different vulnerability relations on the resulting risk mapping will be explored.

The hazard component of risk (Table l) is the main focus of this study. So far, this component has been derived by statistical methods, fitting a statistical distribution to historical avalanche events of known runout distances in a given avalanche path. Because of the usual lack of a sufficient number of recorded events, some authors tried to circumvent the problem by standardizing records from several different avalanche paths to produce a combined artificial history for an average avalanche path within a mountain region. Avalanche events have been transferred between paths either by topographical criteria (Keylock and others, 1999) or by using physical models (Jónasson and others, 1999). However, the main problem with statistical approaches remains to be solved, which is to extrapolate the empirical probability distribution fitted to field data in order to estimate the avalanche frequency in areas not covered by historical information.

In this paper, we introduce a new methodology to estimate the hazard component of avalanche risk. The probability $P_{x, y}(v)$ that a given velocity $(v)$ will be reached or exceeded at any given point $(x, y)$ of the avalanche path is determined by:

$$
P_{x, y}(v)=\int_{0}^{\infty} P_{v ; x, y}^{*}(V) f(V) \mathrm{d} V,
$$

where $f(V)$ is the probability density function (PDF) of the avalanche release volume $V$, and $P_{v ; x, y}^{*}(V)$ is the probability that the avalanche velocity will exceed $v$ at the location $(x, y)$ if the avalanche release volume is $V$. This latter probability is calculated by avalanche dynamics simulations. Risk at a given location $(x, y)$ is then calculated as:

$$
R(x, y)=\int_{0}^{\infty} p_{x, y}(v) d(v) \mathrm{d} v
$$

where $d(v)$ is the vulnerability relation and $p_{x, y}(v)$ is the PDF of avalanche velocity at the location $(x, y)$, obtained by derivation from $Q_{x, y}(v)=1-P_{x, y}(v)$, with $P_{x, y}(v)$ defined in Equation (2). The specific methods introduced to determine the function $P_{x, y}(v)$ and $d(v)$ are described in sections 2 and 3, respectively. In section 4 an application of the procedure to a real-world mapping problem is presented. The potential of the proposed approach for evaluating the residual risk after the implementation of defensive structural work is also shown.

\section{THE HAZARD GOMPONENT OF RISK, $\boldsymbol{P}_{x, y}(v)$}

The probability that a given point along a slope will be reached by an avalanche with a velocity equal to or higher than a fixed value $v$ can be evaluated in principle by considering the release probability of a snow volume $V$ and the probability that the given point along the avalanche path will be reached by an avalanche with a velocity not lower than $v$, given the avalanche release volume is $V$. If the avalanche dynamics is simulated by means of a one-dimensional mathematical model, the computational domain can be represented in a Cartesian plot $(x, z)$, where $x$ is the progressive distance from the fracture line and $z$ is the elevation. In this way, each point on the slope can be identified by means of the $x$ coordinate (so that in Equations (2) and (3) the pair $x, y$ reduces to the coordinate $x$ alone). Within this simplified one-dimensional scheme, the avalanche-release conditions reduce to slab length and fracture depth. Assuming for the slab length a deterministic pattern dependent on the release zone topography and not related to the avalanche frequency at all, the release volume $V$ in Equation (2) will only depend on the release depth $h$.

Introducing a random variable $H$ such that:

$$
H=\left\{\begin{array}{l}
1 \quad \begin{array}{l}
\text { if the avalanche reaches point } x \\
\text { with a velocity } \geq v
\end{array} \\
0 \quad \begin{array}{l}
\text { if the avalanche reaches point } x \\
\text { with a velocity }<v
\end{array}
\end{array}\right.
$$

the probability $P$ that the point $x$ in the runout zone will be reached by an avalanche with a velocity $\geq v(H=1)$ can be expressed as:

$$
P(H=1)=P_{x}(v)=\int_{0}^{\infty} P_{v ; x}^{*}(h) f(h) \mathrm{d} h,
$$

where $f(h)$ is the PDF of the release depth $h$ and $P_{v ; x}^{*}(h)$ is the probability that at point $x$ the avalanche velocity will exceed $v$ given a release depth equal to $h$. Equation (4) is based upon composed and total probability axioms, under the assumption that $P_{v ; x}^{*}(h)$ and $f(h)$ are unrelated. A similar definition has been proposed by the U.S. Federal Emergency Management Agency (FEMA, 1990) for mapping areas affected by debris flows.

The function $f(h)$ is estimated by statistical inference from snowfall data records (see section 2.1), whereas the probability $P_{v ; x}^{*}(h)$ is determined by avalanche dynamics modelling (see section 2.2). Because the avalanche dynamic behaviour must be explored over a wide range of release depths (Equation (4)), the dependence of the model parameters on the avalanche size is crucial (see section 2.3).

\subsection{Estimation of the release-depth PDF, $f(h)$}

According to Salm and others (1990) and Burkard and Salm (1992), the release or fracture depth $h$ can be expressed as a function of the 3 day increase in snow depth $\Delta H(3 \mathrm{~d})$, the 
additional snowdrift depth $\Delta H_{\text {sd }}$ and the average inclination of the release zone $\theta$ as follows:

$$
h=\left(\Delta H(3 \mathrm{~d})+\Delta H_{\mathrm{sd}}\right) s(\theta),
$$

where $s(\theta)$ is a slope factor which takes into account that the release depth decreases with increasing slope angle (Salm and others, 1990). Assuming that $\Delta H_{\text {sd }}$ can be characterized by an estimated average value (Salm and others, 1990), $\Delta H(3 \mathrm{~d})$ is the only random variable in Equation (5). Given the linear relationship between $h$ and $\Delta H(3 \mathrm{~d})$, the following relation holds between their PDFs:

$$
f(h)=f(\Delta H(3 \mathrm{~d})) \frac{1}{s(\theta)} .
$$

The PDF of $\Delta H(3 \mathrm{~d}), f \Delta H(3 \mathrm{~d})$, can simply be inferred by statistical analysis, i.e. by fitting a representative sample of maximum annual values of $\Delta H(3 \mathrm{~d})$ with a theoretical distribution function that shows a good adaptation to the data. Due to the usual lack of snowfall data and to the spatial randomness of $\Delta H(3 \mathrm{~d})$ - meteorological gauging stations are mostly located at lower altitude than the release zones "regional analysis" techniques (Kite, 1988; Cunnane, 1989) provide an effective way of more accurately estimating the PDF of $\Delta H(3 \mathrm{~d})$ (Barbolini and others, 2002), and have been used in the present study.

\subsection{Estimation of the probability $\boldsymbol{P}_{v ; x}^{*}(\boldsymbol{h})$}

To simulate the avalanche propagation and to evaluate the probability $P_{v ; x}^{*}(h)$, VARAlD, the one-dimensional version of the VARA avalanche dynamics models (Natale and others, 1994; Barbolini, 1998; Barbolini and others, 2000; Barbolini and Savi, 2001), has been used. Given that the avalanche release depth is $h$, the avalanche dynamics is simulated with the VARAlD model: $P_{v ; x}^{*}(h)$ is set to 1 if the avalanche reaches point $x$ with a velocity $>v$; otherwise it is set to zero.

\subsection{Dynamic model calibration}

The VARAlD model has been calibrated reproducing historical events from three different Italian alpine ranges: Val di Rabbi (Province of Trento), Val Brembana (Province of Bergamo) and Valtellina (Province of Sondrio). Validation data included the longitudinal profile of the path, the upper and lower limits of the release area, the fracture depth, the runout distance and sometimes some rough information about the deposition height. We calibrated the model by identifying the values of the friction parameters $\mu$ and $\xi$ that minimize the differences between computed and observed runout distance. Further information is required to avoid over-parameterization of the model, since the same runout distance can be obtained using different combinations of the values of $\mu$ and $\xi$. If some information on the spatial distribution of the avalanche deposits was known, the values of the parameters were estimated by minimizing the mean square error between observed and computed runout distance and maximum deposition height. If no information other than the runout distance was available, we reduced the range of admissible values of $\mu$ and $\xi$ by imposing limits on the maximum avalanche velocity, according to measurements from Roger's Pass, British Columbia, Canada by McClung and Schaerer (1993, fig. 5.32).

We found a significant decrease in $\mu$ as the avalanche frequency decreases (and as the release depth $h$ increases); this

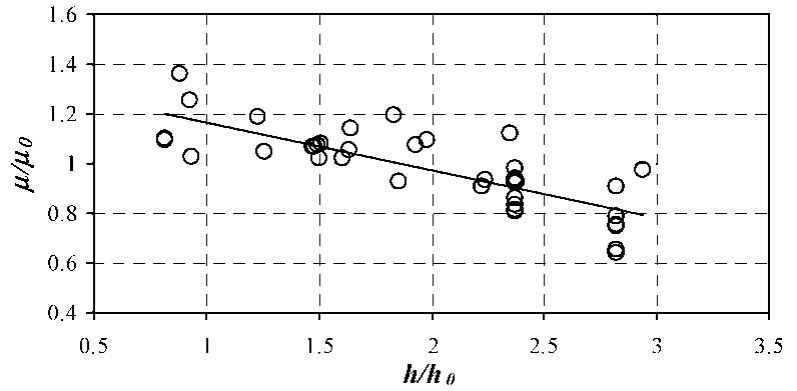

Fig. 1. Normalized friction coefficient $\mu / \mu_{0}$ vs normalized release depth $h / h_{0}$ for 39 calibration events in 17 avalanche paths. The regression line (Equation (7)) has $R=0.79$ and $\sigma=0.095$, with $R$ coefficient of correlation and $\sigma$ standard error of estimate.

trend is also suggested in the Swiss Guidelines (Salm and others, 1990). However, it seems reasonable to introduce a relationship between $\mu$ and $h$ of the type $\mu=F(h)$ only for a specific avalanche site, since the same release depth can be either extreme or very frequent for two different sites. For this reason, the values of $\mu$ and $h$ must be scaled by considering average site-specific values, and a relationship of the type $\mu / \mu_{0}=F\left(h / h_{0}\right)$ must be searched. On the basis of the model calibration, we identified a set of pairs $\mu_{i, j}$ and $h_{i, j}\left(j=1, \ldots, M\right.$ and $i=1, \ldots, N_{j}$ with $M$ the number of sites and $N_{j}$ the number of the calibration events available for the $j$ th site). These values are scaled for each site with the respective average value of $\mu\left(\mu_{0 j}\right)$ and mean annual release depth $\left(h_{0 j}\right)$, to obtain the set of pairs $\left(\mu_{i, j} / \mu_{0 j} ; h_{i, j} / h_{0 j}\right)$, which are plotted in Figure 1. The dimensionless value of the friction coefficient $\mu$ was found to be significantly related to the dimensionless release depth (the $t$ test for the coefficient of correlation, $R=0.79$, is verified with $P=0.01$ ). A least-squares regression gives:

$$
\frac{\mu}{\mu_{0}}=-0.193 \frac{h}{h_{0}}+1.361 \text {. }
$$

For a given avalanche site, once the values of $\mu_{0}$ and $h_{0}$ have been estimated, Equation (7) gives the site-specific relation between $\mu$ and $h$, which, in turn, once a typical release area is defined, allows the site-specific relationship between $\mu$ and the release volume $V$ to be defined.

\section{THE VULNERABILITY COMPONENT OF RISK, $d(v)$}

Jónasson and others (1999) linked avalanche speed to survival probability inside a building by a maximum likelihood technique. Their analysis was based on data from the 1995 Icelandic avalanches at Súdavik and Flateyri, which damaged a total of 32 houses where 93 people were staying, causing 34 fatalities. They obtained the following vulnerability relation $d(v)$, indicating the probability of death inside a house as a function of the avalanche velocity:

$$
d(v)= \begin{cases}k v^{2} & \text { if } v<v_{1} \\ 1-c-\frac{a}{v-b} & \text { if } v \geq v_{1}\end{cases}
$$

where $k=0.00130, c=0.05, a=1.151, b=18.61$ and $v_{1}=$ $23 \mathrm{~m} \mathrm{~s}^{-1}$ (Fig. 2). The vulnerability decreases progressively to zero with decreasing avalanche velocity; there is an upper limit of 0.95 if the avalanche velocity becomes indefinitely 


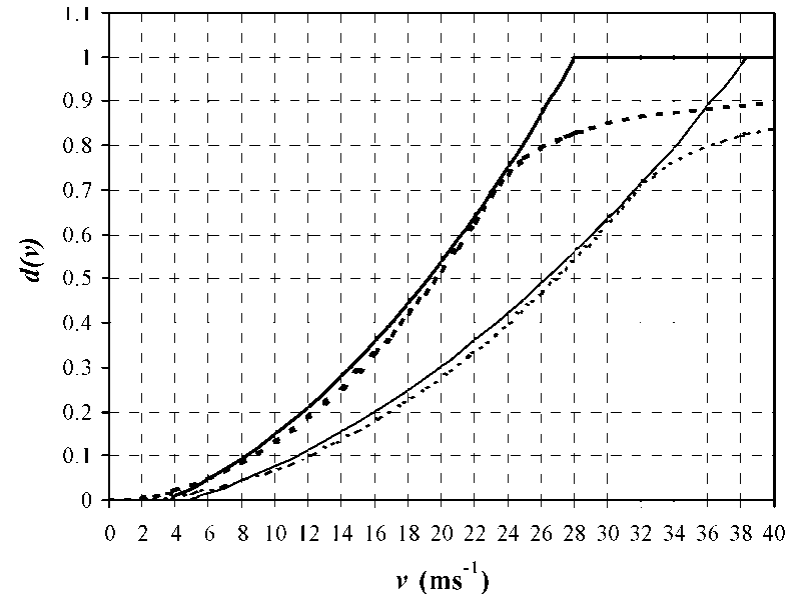

Fig. 2. Vulnerability vs avalanche velocity. The dashed bold line shows the relation proposed by fónasson and others (1999) (Equation (8)); the solid bold line shows the relation including velocity thresholds derived in this study based on Icelandic data (Equation (9)). The other two curves represent the vulnerability relations for (Icelandic) reinforced structure for the case with (solid) and without (dashed) thresholds.

high. It is therefore implicitly assumed that a loss of life is always possible with a non-zero velocity and that, regardless of how fast the avalanche travels, some people will always survive.

However, it seems reasonable to assume that people are safe inside a building for low-intensity events. Therefore it is proposed to introduce a lower threshold for the velocity, $v_{\text {lt }}$, below which vulnerability can be set to zero. This is in accordance with the proposal of Keylock and others (1999) who indicate zero vulnerability for the smallest avalanche size ( 1 and 2 according to the Canadian Avalanche Size Classification, with typical impact pressure in the range $1-10 \mathrm{kPa}$; see Keylock and others, 1999, tables 1 and 9). Keylock and Barbolini (2001) presented a vulnerability relation which also contains an upper threshold for the velocity, $v_{\text {ut }}$, which was estimated to be about $15 \mathrm{~m} \mathrm{~s}^{-1}$ for Icelandic types of building. For velocity

Table 2. Dependence of death rate d on avalanche velocities v with respect to the 1995 avalanche events in Súdavik and Flateyri, Iceland

\begin{tabular}{ccc}
\hline $\begin{array}{c}\text { Velocity class } \\
\left(\mathrm{m} \mathrm{s}^{-1}\right)\end{array}$ & $\begin{array}{c}\text { Reference velocity } \\
\left(\mathrm{m} \mathrm{s}^{-1}\right)\end{array}$ & $d(v)$ \\
\hline $0-5$ & 2.5 & 0 \\
$5-10$ & 7.5 & 0 \\
$10-15$ & 13.6 & 0.54 \\
$15-20$ & 17.2 & 0.13 \\
$20-25$ & 22.0 & 0.74 \\
$25-30$ & 26.5 & 0.9 \\
\hline
\end{tabular}

Notes: Data are taken from Jónasson and others (1999). $d(v)$ is calculated for each velocity class as ratio between the total number of people at home and the total number of people killed; avalanche velocities have been estimated with the PROTÉON/Crocus/MÉPRA (PCM) model by Jónasson and others (1999). The velocity classes are taken to be $5 \mathrm{~m} \mathrm{~s}^{-1}$ wide, but in deriving Equation (9) the average vulnerability for each class is related to a reference velocity that represents the average of the observed velocity for each class. Merging classes " $10-15$ " and " $15-20$ " does not result in a substantial modification of the best-fitting curve (Equation (9)) as well as using a classification based on wider velocity classes (8 and $10 \mathrm{~m} \mathrm{~s}^{-1}$ wide, respectively). values higher than $v_{\text {ut }}$ the vulnerability was set to 1 . The existence of such a threshold is more questionable, given that even the complete destruction of a building does not necessarily entail the death of a person staying inside. Wilhelm (1998) presented plots of the susceptibility to damage $(S)$ as a function of impact pressure for different types of construction ( $S$ varies between 0 (no damage) and 1 (house destruction)). All these curves showed a destruction limit (varying between 10 and $50 \mathrm{kPa}$ depending on the construction type). However, a probability of death $<1$ for people inside a destroyed house was suggested. A similar result can be derived from the vulnerability data presented in Keylock and others (1999).

In order to explore the effect on the risk mapping of introducing thresholds in the vulnerability relation, we have reworked the Icelandic data (Jónasson and others, 1999, table 7), to obtain a vulnerability relation with thresholds. This curve, derived by fitting a second-order polynomial to the data of Table 2, is presented in Figure 2 and has the following form:

$$
\left\{\begin{array}{rlrl}
d(v)=0 & & \text { if } v \leq 3.5 \mathrm{~m} \mathrm{~s}^{-1} \\
d(v)=0.001 v^{2}+0.0091 v & & \\
& -0.0426 & & \text { if } 3.5<v<28 \mathrm{~m} \mathrm{~s}^{-1} \\
d(v)=1 & & \text { if } v \geq 28 \mathrm{~m} \mathrm{~s}^{-1}
\end{array}\right.
$$

The lower velocity threshold is $3.5 \mathrm{~m} \mathrm{~s}^{-1}$, corresponding to an impact pressure of about $3 \mathrm{kPa}$ according to Equation (1). This seems to be a reasonable value, given that Wilhelm (1998) suggests a value of about $2.5 \mathrm{kPa}$ as the building damage threshold for the lowest quality of construction ("light construction"). The upper velocity threshold gives an impact pressure of about $250 \mathrm{kPa}$ (see Equation (1)), a value about an order of magnitude higher than the impact pressure that McClung and Schaerer (1993) indicate can destroy a wood frame house $(30 \mathrm{kPa})$. This partly justifies our first estimate of the threshold for $100 \%$ fatalities within an unreinforced structure with poorly supported walls, as is the case for Icelandic houses.

Both vulnerability relations (Equations (8) and (9)) are derived from Icelandic data. It should be pointed out that most of the houses in the avalanche hazard zones of Iceland are fairly weak, made of timber or unreinforced concrete, with relatively large windows facing the mountain slope. Typical buildings in Alpine villages are suggested to be less vulnerable, and using Equation (8) or (9) might overestimate the actual risk levels. Using earthquake data, Keylock and others (1999) suggested that for reinforced concrete structures vulnerability values can be assumed to be about $60 \%$ of those for low-quality buildings. Applying this ratio to our case, the residual vulnerability in Equation (8) decreases from 0.95 to 0.57 , whereas the upper and lower velocity thresholds remain unchanged in Equation (9). In fact, reinforcing a structure should lead to an increase of both $v_{\text {ut }}$ and $v_{\text {lt }}$. Nevertheless, the residual vulnerability associated with a random chance of survival independent of avalanche intensity should not be influenced by structural reinforcement. Re-analyzing the data presented by Keylock and others (1999), we have obtained an average rate of velocity increase of about 1.37 which leads, after reinforcement, to the same vulnerability value as before. Using this value to correct Equations (8) and (9), we have obtained the vulnerability relations for (Icelandic) reinforced structures, presented in Figure 2 for the case without and with 


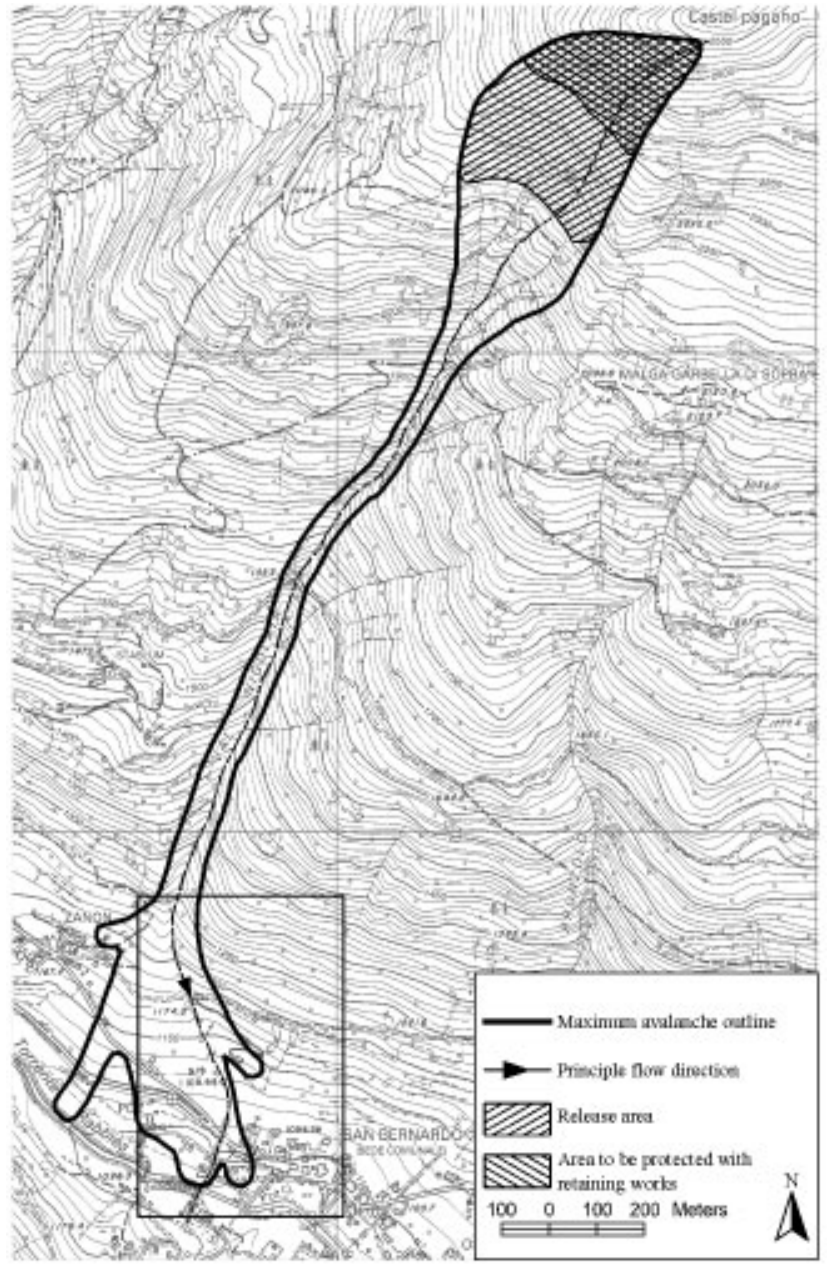

Fig. 3. Map of the avalanche site Val Nigolaia. The runout area, indicated by the rectangle, is shown in Figure 7.

thresholds, respectively. We may tentatively assume the vulnerability of Alpine buildings lies between the reinforced and non-reinforced Icelandic cases.

\section{GASE STUDY}

\subsection{Description of the site}

The methodology previously described is applied to the Val Nigolaia avalanche site, a path located in the Italian Alps, within the Autonomous Province of Trento. Val Nigolaia is a tributary valley of Val di Rabbi that originates from the Castel Pagano top crest, at about $2600 \mathrm{~m}$ a.s.l. The site is mainly channelled and covers a vertical drop of about $1500 \mathrm{~m}$ (Fig. 3); its length is about $3 \mathrm{~km}$. The release area, with a south-southwest aspect, is situated at approximately 2550-2200 m a.s.l. and covers an area of about $0.095 \mathrm{~km}^{2}$, with an inclination of $35-45^{\circ}$. The track is constituted by a very narrow channel (about 10-15 m wide); its slope gradually decreases from about $30^{\circ}$ in the upper part to about $20^{\circ}$ in the lower part. The track terminates at about $1250 \mathrm{~m}$ a.s.l., where the deposition zone begins, represented by a fairly large alluvial fan. In the deposition zone, the slope gradually decreases from $15^{\circ}$ in the upper part of the fan to a nearly flat zone close to the Rabbies river. The biggest known event on this path occurred in 1931: a huge wet-snow avalanche affected the western part of S. Bernardo village,

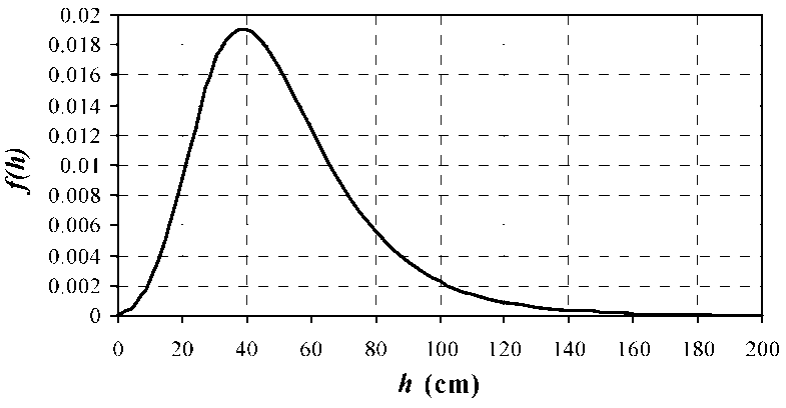

Fig. 4. PDF of the release depth $h$ for the Val Nigolaia path, obtained by "regional analysis" of annual maximum of the snow-depth increase over 3 days, $\Delta H(3 \mathrm{~d})$ (eight gauging stations for a total of 158 annual maxima). The regional data have been fitted to a generalized extreme values (GEV) distribution, with parameters estimated using the averageweighted-moments technique.

damaging some buildings and depositing snow masses down to the Rabbies river (Fig. 3).

\subsection{Application of the methodology}

In the simulations, the release length $L$ is held constant and set to $L=500 \mathrm{~m}$. The upper limit of the release area is fixed at $2550 \mathrm{~m}$ a.s.l., according to chronicle information. The friction coefficient $\xi$ is set to $5900 \mathrm{~m} \mathrm{~s}^{-2}$ (the mean of the calibrated values over the Val Nigolaia site) and kept constant in all the simulations, in accordance with the Swiss Guidelines (Salm and others, 1990) which suggest that this parameter is mainly influenced by the degree of canalization and roughness of the track and not by the avalanche size. The average calibration value of the friction coefficient $\xi$ may seem to be surprisingly high if compared to the reference values proposed in the Swiss Guidelines (Salm and others, 1990), but this relates to a general tendency of "hydraulic-type" dynamic models to require larger values of $\xi$ than "centre-of-mass-type" dynamic models (Barbolini and others, 2000). The friction coefficient $\mu$ is varied with the fracture depth according to Equation (7).

$P_{x}(v)$ for any given position $x$ of the runout area was estimated for 60 different velocities, from 0.5 to $30 \mathrm{~m} \mathrm{~s}^{-1}$ with an increment step of $0.5 \mathrm{~m} \mathrm{~s}^{-1}$. Velocities higher than $30 \mathrm{~m} \mathrm{~s}^{-1}$ are never obtained below $1250 \mathrm{~m}$ a.s.l., where risk calculations are of interest. A position $x$ and a velocity threshold $v$ having been fixed, $P_{x}(v)$ is estimated by numerical integration of Equation (4), which reads:

$$
P_{x}(v)=\sum_{i=1}^{n} P_{v ; x}^{*}\left(h_{i}\right) f\left(h_{i}\right) \Delta h_{i}
$$

and requires the following steps: a value of the fracture depth $h_{i}$ is assigned; the value of $f\left(h_{i}\right)$ is evaluated from the PDF of $h$, shown in Figure 4 for the site under analysis; the value of $\mu_{i}$ is computed by means of Equation (7), adopting $h_{0}=0.5 \mathrm{~m}$ and $\mu_{0}=0.26$ (estimated average values of the release depth $h$ and of the friction coefficient $\mu$ for the site under analysis); the propagation of the $i$ th avalanche is simulated by means of the VARAlD code; if the considered point $(x)$ of the computational grid is reached by the avalanche with a velocity greater than $v \mathrm{~ms}^{-1}$, then $P_{v ; x}^{*}\left(h_{i}\right)=1$, otherwise $P_{v ; x}^{*}\left(h_{i}\right)=0$. The above steps are repeated 40 times for each considered velocity threshold (we varied $h$ from $0.1 \mathrm{~m}$ to $2 \mathrm{~m}$, with an increment of 


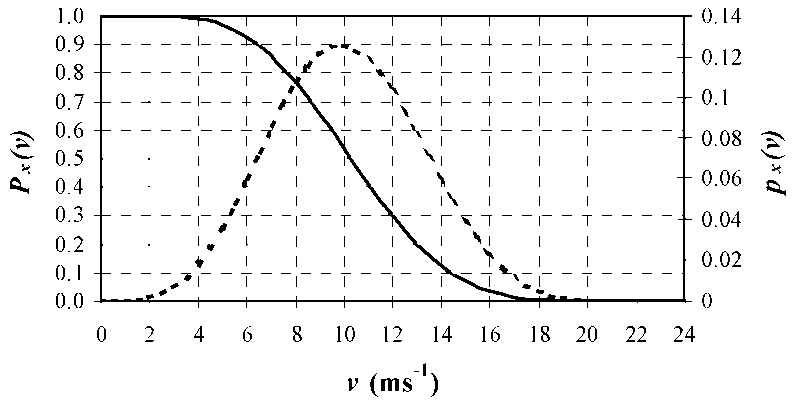

Fig. 5. Probability that the avalanche velocity exceeds $v$ at the location $x, P_{x}(v)$ (solid line), and PDF of the avalanche velocity $v$ at the location $x, p_{x}(v)$ (dotted line), relative to the position along the slope given by $x=2200 \mathrm{~m}$, corresponding to an altitude of $1220 \mathrm{~m}$ a.s.l. (the fan apex, approximately).

$0.05 \mathrm{~m}$ ), the values of the summation terms in Equation (10) are evaluated, and the probability $P_{x}(v)$ can be calculated for the selected values of velocity. A typical result of this computation is shown in Figure 5. $p_{x}(v)$ is obtained by numerical derivation of $Q_{x}(v)=1-P_{x}(v)$ (see Fig. 5). Of course, both $P_{x}(v)$ and $p_{x}(v)$ change as the position $x$ along the path changes.

To obtain risk, Equation (3) is solved numerically:

$$
R(x)=\sum_{i=1}^{n} p_{x}\left(v_{i}\right) \mathrm{d}\left(v_{i}\right) \mathrm{d} v .
$$

To solve Equation (11) for any given location $x$, it is necessary to fix a velocity value $v_{i}$; to estimate $p_{x}\left(v_{i}\right)$ from the PDF $p_{x}(v)$ relative to the selected location (see Fig. 5 for the location $x=2200 \mathrm{~m})$; and to estimate $d\left(v_{i}\right)$ from the chosen vulnerability relation. The previous steps are repeated 60 times, varying $v$ from 0.5 to $30 \mathrm{~m} \mathrm{~s}^{-1}$, with an increment step of $0.5 \mathrm{~m} \mathrm{~s}^{-1}$. The risk was estimated using the different vulnerability relations presented in Figure 2. Risk was also estimated assuming that part of the release zone is protected by snow bridges (about $40 \%$ of the potential release area, from 2550 to $2350 \mathrm{~m}$ a.s.l.; see Fig. 3). In this latter

Table 3. Risk calculation in the runout area for different modelling assumptions

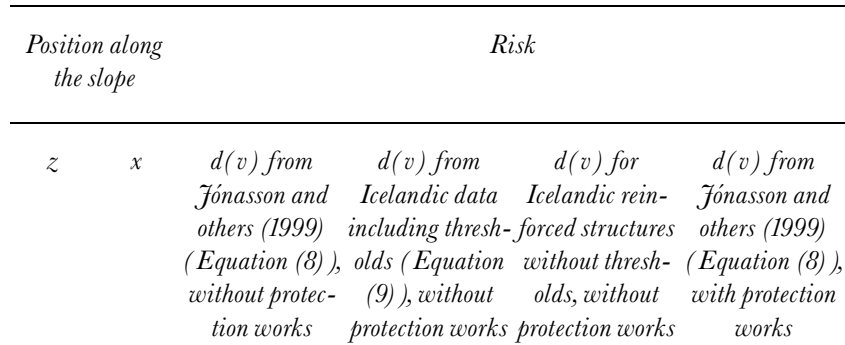

ma.s.l. $\mathrm{m}$

\begin{tabular}{cccccc}
\hline 1200 & 2260 & $1.0 \times 10^{-1}$ & $1.1 \times 10^{-1}$ & $5.4 \times 10^{-2}$ & $6.1 \times 10^{-2}$ \\
1150 & 2425 & $2.8 \times 10^{-2}$ & $2.9 \times 10^{-2}$ & $1.5 \times 10^{-2}$ & $8.6 \times 10^{-3}$ \\
1130 & 2515 & $6.1 \times 10^{3}$ & $6.2 \times 10^{-3}$ & $3.3 \times 10^{-3}$ & $1.4 \times 10^{-3}$ \\
1120 & 2565 & $3.3 \times 10^{-3}$ & $3.3 \times 10^{-3}$ & $1.7 \times 10^{-3}$ & $3.1 \times 10^{-4}$ \\
1110 & 2615 & $1.9 \times 10^{-3}$ & $1.9 \times 10^{-3}$ & $1.0 \times 10^{-3}$ & $8.5 \times 10^{-5}$ \\
1105 & 2645 & $1.1 \times 10^{-3}$ & $1.1 \times 10^{-3}$ & $6.1 \times 10^{-4}$ & $4.0 \times 10^{-5}$ \\
1100 & 2675 & $7.5 \times 10^{-4}$ & $6.9 \times 10^{-4}$ & $4.0 \times 10^{-4}$ & $9.7 \times 10^{-6}$ \\
1095 & 2715 & $3.1 \times 10^{-4}$ & $2.3 \times 10^{-4}$ & $1.7 \times 10^{-4}$ & 0 \\
1090 & 2760 & $1.4 \times 10^{-4}$ & $4.9 \times 10^{-5}$ & $7.2 \times 10^{-5}$ & 0 \\
& & & & & \\
\hline
\end{tabular}

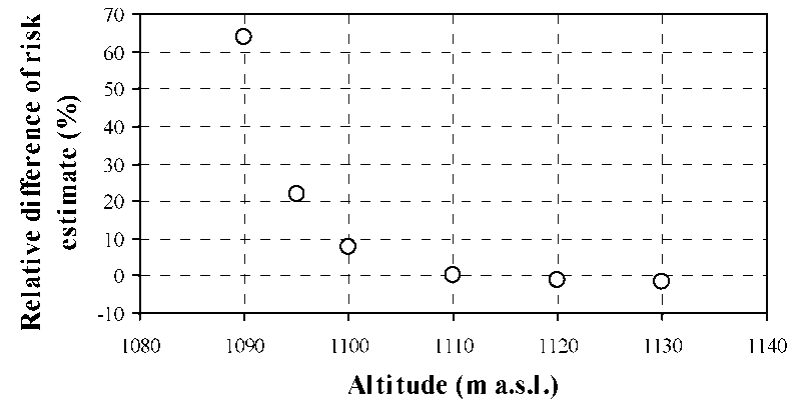

Fig. 6. Difference between risk estimates based on vulnerability Equation (8), taken from fónasson and others (1999), and Equation (9), derived in this study based on Icelandic data and including upper and lower velocity thresholds, as a function of location along the path (expressed in $m$ a.s.l.).

case, the release scenarios and the friction parameters were adjusted accordingly.

\subsection{Results}

Results of the simulations are summarized in Table 3. Using the vulnerability relation given by Jónasson and others (1999) (Equation (8)), we obtain risk values ranging from about $10^{-1}$ to $1.4 \times 10^{-4}$ going from the upper part (fan apex, $z=1200 \mathrm{~m}$ a.s.l.) to the lower part (river location, $z=$ $1090 \mathrm{~m}$ a.s.l.) of the runout area. Use of a vulnerability relation that includes thresholds (Equation (9)) leads to

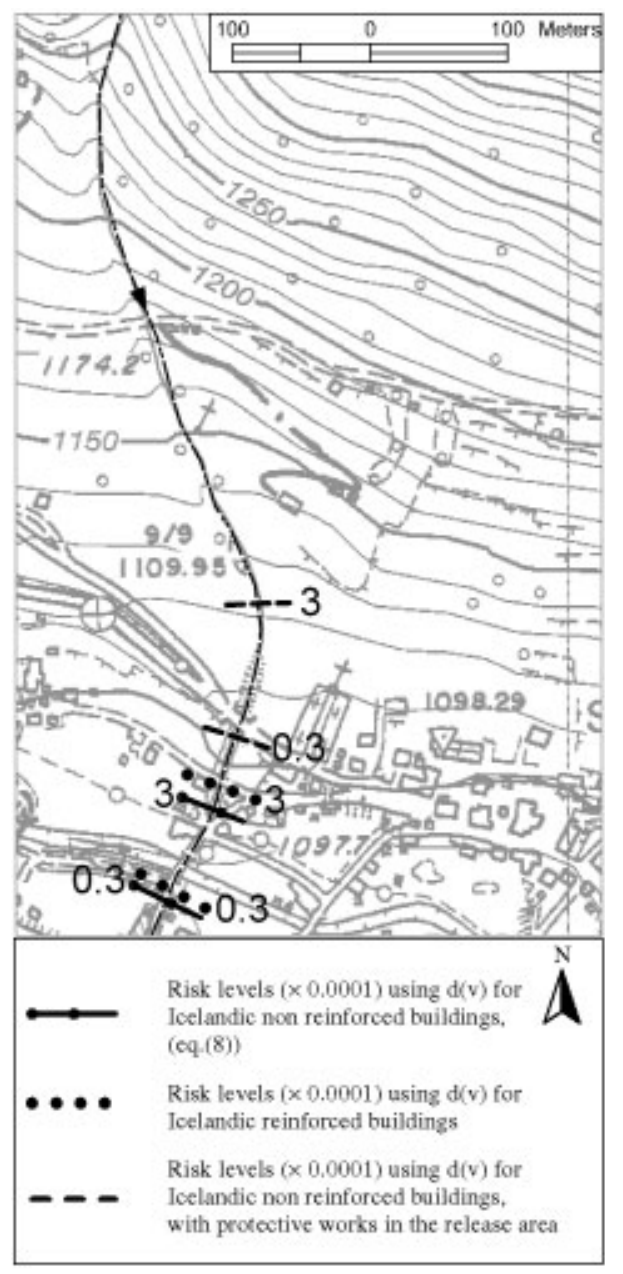

Fig. 7. Risk mapping according to different modelling assumptions. 
smaller risk values in the lower part of the runout zone (in particular in the area below the provincial road, i.e. for $z<$ $1110 \mathrm{~m}$ a.s.l.), with maximum differences up to $60 \%$ (Fig. 6). In fact, introducing a lower velocity threshold in the vulnerability relation reduces the risk level at smaller velocities, i.e. in the terminal part of the deposition zone. For the considered case, $3 \times 10^{-4}$ and $1 \times 10^{-4}$ risk lines are moved upslope by about 10 and $25 \mathrm{~m}$, respectively.

Use of the vulnerability relation for reinforced structures, of course, leads to lower risk values throughout the runout area (see Table 3). Differences with respect to the non-reinforced building case are of the order of about $45 \%$ for all the locations in the deposition zone. Assuming a relevant part of the release area is protected with retaining structures (see Fig. 3) leads to considerably reduced risk values in the runout area (Table 3). In particular, the risk approaches zero below $1100 \mathrm{~m}$ a.s.l.

In Figure 7, the positions of the calculated risk levels $3 \times 10^{-4}$ and $0.3 \times 10^{-4}$ are indicated for three different modelling assumptions. These two risk levels are relevant for hazard mapping according to the Icelandic regulation (Jóhannesson and Arnalds, 2001). In particular, a calculated risk below $0.3 \times 10^{-4}$ is considered acceptable for any kind of land use, whereas major restrictions on land use are applied if the risk is $>3 \times 10^{-4}$. For the site under analysis, the risk levels appear to be unacceptably high in the inhabited areas located at the valley bottom (Fig. 7), even if the average value lies between the reinforced and non-reinforced Icelandic cases, which seems a reasonable assumption for Alpine buildings. The protection of part of the release area with retaining structures considerably improves the safety of the population living in the endangered areas, and acceptable risk levels are attained over most of S. Bernardo village.

\section{CONGLUDING REMARKS}

A new method for estimating risk in avalanche-prone areas has been proposed. Risk is defined as the yearly probability of being killed by an avalanche if one lives or works permanently in a building under a hazardous slope. Vulnerability, expressing the probability of death, is given as a function of the velocity of the avalanche hitting the building, according to previous formulations. The main innovation with respect to the current approaches is the use of an avalanche-dynamics model to estimate the hazard component of risk, i.e. the probability of having a given velocity for any fixed location along the avalanche path. So far the methodology has been implemented in a one-dimensional framework. However, extension to two dimensions is in principle quite straightforward, and simply requires the use of a two-dimensional dynamic model. The use of dynamic models has the twofold advantage of: (i) avoiding the problem of statistical inference from runout data, which in principle is inappropriate for extrapolations to undocumented areas; and (ii) estimating systematically the residual risk after the implementation of defence works. Accordingly, the method could provide a sound base for cost-benefit evaluations.

A relevant limit of our procedure is the lack of knowledge of vulnerability, i.e. how avalanche impact damages structures and causes fatalities. A fatality ratio which is a function of the avalanche velocity alone, such as the one we have used, is of course an oversimplification. More complex formulations that consider other dynamical parameters than velocity (e.g. the flow depth) will improve the method significantly. Furthermore, so far we have used relations based on Icelandic data. Specific studies are needed to define vulnerability relations more suitable for typical Alpine buildings. The possible existence of velocity thresholds in the vulnerability relation, as well as their effect on risk mapping, is another point that requires further investigation.

The uncertainties affecting the application of the dynamic model (related to either release conditions or friction-parameters estimate), as well as those underlying the definition of the vulnerability relation, can easily be incorporated into the methodology by using Monte Carlo simulation techniques, according to recent proposals (Barbolini and Savi, 2001; Barbolini and others, 2002). This point will be addressed as the most immediate follow-up to this work.

\section{ACKNOWLEDGEMENTS}

This research has been financially supported by the National Group for the Prevention of Hydrogeological Disasters (GNDCI) of the National Research Council of Italy (CNR). The authors wish to gratefully acknowledge L. Natale for his guidance in the development of this work, and $\mathrm{G}$. Tecilla, M. Gaddo and G. Fruet from the Avalanche Office of the Autonomous Province of Trento for supporting the data collection.

\section{REFERENGES}

Barbolini, M. 1998. VARA one- and two-dimensional models. In Harbitz, C.B., ed. EU Programme SAME. A Survey of computational models for snow avalanche motion. Oslo, Norwegian Geotechnical Institute, 59-63. (NGI Report No. 581220-1.)

Barbolini M. and F. Savi. 2001. Estimate of uncertainties in avalanche hazard mapping. Ann. Glaciol., 32, 299-305.

Barbolini, M., U. Gruber, C. J. Keyloch, M. Naaim and F. Savi. 2000. Application and evaluation of statistical and hydraulic-continuum densesnow avalanche models to five real European sites. Cold Reg. Sci. Technol., 31 (2), 133-149.

Barbolini M., L. Natale and F. Savi. 2002. Effect of release condition uncertainty in avalanche hazard mapping. Natural Hazards, 25(3) 225-244.

Bozhinsky, A. N. and K.S. Losev. 1998. The fundamentals of avalanche science. Eidg. Inst. Schnee- und Lawinenforsch. Mitt., 55.

Burkard, A. and B. Salm. 1992. Die Bestimmung der mittleren Anrissmächtigkeit $d_{0}$ zur Berechnung von Fliesslawinen. Davos, Swiss Federal Institute for Snow and Avalanche Research. (Internal Report 688.)

Cunanne, C. 1989. Statistical distributions for flood frequency analysis. Geneva, World Metereological Organization. (Operational Hydrology Report 33.)

Evans, A.W., P. B. Foot, S. M. Mason, I. G. Parker and K. Slater. 1997. Third party risk near airports and public safety zone policy. London, National Air Traffic Services Ltd.

F.E.M.A. 1990. Fan: an alluvial flooding computer program. Washington, DC, Federal Emergency Management Agency.

IUGS (International Union of Geological Sciences) Working Group on Landslides, Committee on Risk Assessment. 1997. Quantitative risk assessment for slopes and landslides - the state of the art. In Cruden, D.M. and R. Fell, eds. Landslide risk assessment. Proceedings of the International Workshop on Landslide Risk Assessment, 19-21 February 1997, Honolulu, Hawaii, U.S.A. Rotterdam, A.A. Balkema, 3-12.

Jóhannesson, T. and T. Arnalds. 2001. Accidents and economic damage due to snow avalanche and landslide in Iceland. Fökull, 50, 81-94.

Jónasson, K., S.T. Sigurðsson and T. Arnalds. 1999 Estimation of avalanche risk, Reykjavik, Vedurstofu Islands. (Report No. R99001-URO.)

Keylock, C. J. and M. Barbolini. 2001. Snow avalanche impact pressure/ vulnerability relations for use in risk assessment. Can. Geotech. 7., 38, 227-238.

Keylock, C. J., D. M. McClung and M. M. Magnússon. 1999. Avalanche risk mapping by simulation. F. Glaciol., 45(150), 303-314.

Kite, G. W. 1988. Frequency and risk analysis in hydrology. Littleton, CO, Water Resources Publications. 
McClung, D. M. and P. A. Schaerer. 1993. The Avalanche Handbook. Seattle, WA, The Mountaineers.

Mellor, M. 1978. Dynamics of snow avalanches. In Voight, B., ed. Rockslides and avalanches: natural phenomena. Vol.1. Amsterdam, Elsevier Scientific Publishing Co., 753-792.

Natale, L., L. Nettuno and F. Savi. 1994. Numerical simulation of snow dense avalanche: an hydraulic approach. In Hamza M.H., ed. Proceedings of 24th International Conference on Modelling and Simulations, MS'94, 2-4 Mar 1994, Pittsburgh, Pennsylvania. Anaheim, CA, International Association of
Science and Technology Development, Acta Press, 233-236.

Salm, B., A. Burkard and H. Gubler. 1990. Berechnung von Fliesslawinen: eine Anleitung für Praktiker mit Beispielen. Eidg. Inst. Schnee- und Lawinenforsch. Mitt. 47.

Wilhelm, C. 1998. Quantitative risk analysis for evaluation of avalanche protection projects. In Hestness, E., ed. 25 Years of Snow Avalanche Research at NGI,Voss, 12-16 May 1998. Proceedings. Oslo, Norwegian Geotechnical Institute, 288-293. 